\title{
ANN Models to Correlate Structural and Functional Conditions in AC Pavements at Network Level
}

\author{
Fawaz Alharbi ${ }^{1}$, Omar Smadi ${ }^{2}$ \\ ${ }^{1}$ Graduate Student, Institute for Transportation, Iowa State University; 2711 South Loop Drive, Suite 4700, Ames, Iowa \\ ${ }^{2}$ Director; Center for Transportation Research and Education, Institute for Transportation, Iowa State University; 2711 South \\ Loop Drive, Suite 4700, Ames, Iowa
}

\begin{abstract}
Artificial Neural Network (ANN) model was developed to estimate the correlation between structural capacity and functional conditions in Asphalt Cement (AC) pavements at the network level. To achieve this objective, the relevant data were obtained and integrated from the Iowa Pavement Management Program (IPMP) including construction parameters, traffic loading and subgrade stiffness, and Iowa Environmental Mesonet (IEM) for climate data. The ANN model proves its ability to learn and generalize from the input data. Overall, rutting data were found to be appropriate indicator of the structural capacity. Since the deflection tests are expensive and require experience and knowledge to deal with such data, this approach might be feasible for small transportation agencies (cities and counties) that do not have these capabilities.
\end{abstract}

Keywords-Artificial Neural Network, AC pavement, Functional Performance, Iowa pavements, Structural performance.

\section{INTRODUCTION}

State Highway Agencies (SHAs) spends millions of dollars each year on providing and managing transportation infrastructures. Evaluating structural capacity is an important consideration in pavement highway systems to optimize network maintenance and agency fund allocation. Most of structural capacity evaluations have been done at the project level, and many highway agencies do not include structural condition evaluations in their Pavement Management Systems (PMS) at the network level management for many reasons such as costs of conducting structural tests and they require experiences to carry them out. In State of Indiana the routinely structural evaluation and thickness data often are not available for Indian pavement network [9] . Agarwal et al. (2006) reported that more than $75 \%$ of highway agencies in India do not carry out any structural evaluations on pavement conditions [3].
Researchers have proposed a number of methodologies to evaluate the structural capacity of pavements by using nondestructive testing. There are various nondestructive testing equipment that are used for pavement evaluation, and falling weight deflectometer (FWD) is the popular one. The FWD applies loads to the pavement surface and the resulting surface deflections are measured by sensors at different distances from the load source (Fig. 1). AASHTO (1993) provided an accurate approach for determining the Structure Number (SN) using the deflection results from FWD [1].

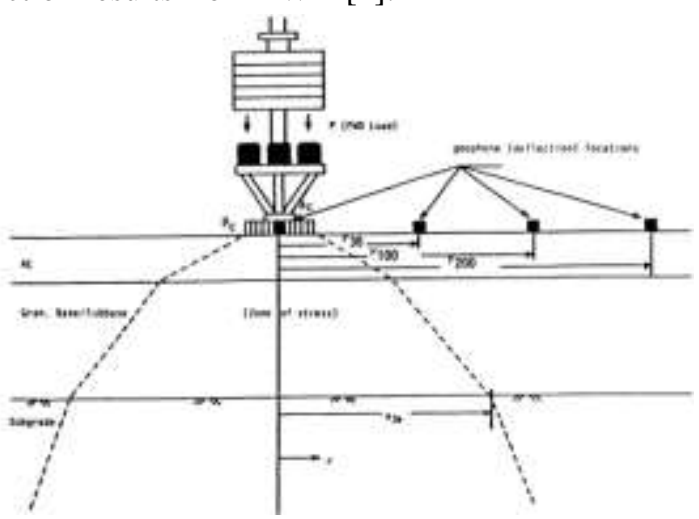

Fig. 1: Schematic of stress zone under the FWD load [12]

However, applying nondestructive tests to provide structural capacity evaluation has many shortcomings because of the FWD stop-and-go operation which affect traffics flow. Moreover, the process of analyzing acquired data is often complex and requires experience and knowledge to deal with such data [3].

Many studies have tried to evaluate the correlation between roughness and structural capacity of pavements at the project level. Sollazzo et al. (2017) conducted ANN models with high accuracy to find the relation between roughness and structural performance in AC pavement by using Long Term Pavement Performance (LTPP) data [13]. Agarwal et al. assessed the relation between alligator cracking and rutting, and pavement structural 
conditions [3]. The pavement structural adequacy can be estimated from existing distress and backward calculation to design procedures [1].

ANN models have recently been widely used to simulate the human process in the brain. ANN models use the collected data to build prediction models and compute the relative importance of variables instead of the natural relationship between variables. Rafiq et al. (2001) reported a definition of an ANN as: "A computational mechanism has an ability to acquire, represent and compute mapping from one multivariate space of information to another, given a set of data representing that mapping" [10].

Engineers often find incomplete or noisy data, so the ANN models are the most applicable models to learn and generalize from the input data until meaningful relation are found to problems [10]. The ANN model has an ability to predict the nonlinear relationships between variables as similar to traditional models [16].

ANNs have been widely used in different civil engineering areas with good results because they are very generic, accurate and convenient mathematical models able to simulate numerical model components [8]. Adeli (2001) had reviewed the papers that had used neural network models since 1989 especially in structural engineering, construction engineering, and management [2]. Golshani et al. (2017) compared the prediction capabilities between statistical approach model and neural network models for modeling two critical trip-related decisions of travel mode and departure time [6]. Their results show the neural network model offers better performance with easier and a faster implementation process. Moreover, ANN and multivariable regression models were used to predict the stress intensity factors (SIFs) in pavement cracking, the results show the advantage of utilizing ANN over multivariable regression models on the prediction accuracy [15]. Felker, et al. (2004) used the ANN and statistical analysis approaches to develop the reliable and accurate roughness prediction models for jointed plain concrete pavements, and they found the ANN is able to predict the roughness with reasonably high coefficient of determination, R-squared= 0.90 , whereas R-squared of statistical analysis approach $=0.73$ [4]. Gencel et al. (2011) presented a comparison between ANN and general linear (GL) models to figure out the correlation between cement content, metal content and traffic loading on the rough wear of concrete [5]. The comparison results show the robustness of ANN models compared to the GLM models. Also, Vlahogianni and Karlaftis (2013) compared between ANN and autoregressive time series models for forecasting the freeway speeds, and they found that neural networks provide more accurate predictions than classical statistical approaches [8].

In this paper, the ANN models have trained to find a reliable relation between $\mathrm{SN}$ and rutting at the network level data. The rutting is defined as a deformation on AC layers or subsurface layers [12].

A large data set of input parameters are included in the model to capture the relevant factors such as structure parameters, traffic loading, climate factors, subgrade stiffness, and pavement age. The ANN model output shows satisfactory results which are adequate to pay attention on this relation for improving the pavement management system.

\section{DATA}

In order to have enough data for training the ANN models, the historical database from the Iowa Pavement Management Program (IPMP) was used. The IPMP started in 1994 to develop, implement, and operate a pavement management system on 23,500 miles of roads in Iowa [7]. The database contains records on structural characteristics, maintenance activities, and traffic details. Also, Long-term climate data was obtained from the Iowa Environmental Mesonet (IEM). The Geographic Information System (GIS) was used to relate weather data that is available from point sources to their highway network. The analysis focuses on AC pavement sections that have not been exposed to any maintenance or rehabilitation operations.

The detailed database included in the model is listed below:

1) Pavement age (years): age of pavement since construction, major rehabilitation or overlay date.

2) Pavement thickness (in): including asphalt layers, base and subbase layers.

3) Total asphalt depth (in )

4) Structural Number (SN)

5) Average annual Equivalent signal axle loads (ESALs) (18,000-Ib)

6) Average daily number of trucks

7) Climate data
a) Average Annual Temperature (F)
b) Average annual number of days with snowfall (days).
c) Average annual of rainfall (inches).
d) Freeze-thaw cycles

8) Performance Parameters:

a) Rutting (inches) 


\section{METHODOLGY}

In order to construct the neural network, three components must first be identified: architecture, learning method and neuron activation function.

\subsection{Architecture}

The architecture of neural network includes determining the input layers, hidden layers and output layers (Fig. 2). The challenge with neural network is how many neurons should be in the hidden layers as they impact the model performance. There is no specific method to select the appropriate number of neurons, so trial and error has been used in many studies.

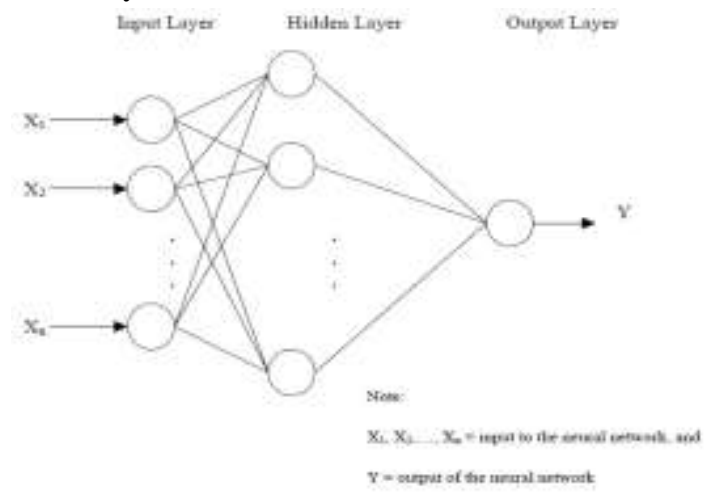

Fig. 2: Neural Network Architecture

\subsection{Learning Method}

The three-layered feed forward back propagation neural network is used during the training process to adjust the weight between the layers. During the training process, the resulted error from the output that is calculated with the initial connection weights are returned back through the hidden layers for many times until the actual and calculated output meet within pre-determined range.

\subsection{Neuron Activation Function}

Each neuron in the hidden layer has own summation and transfer functions with input and output values as illustrated in Fig. 3. The output of summation function is showed in (1).

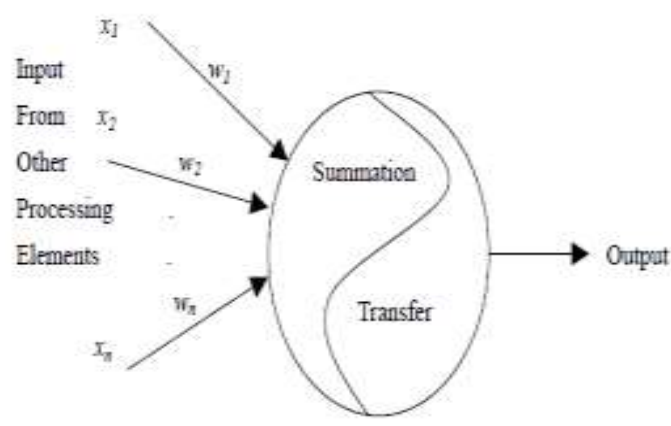

Fig. 3: Diagram of Artificial Neuron

$$
O_{j}=f\left(\sum_{i=1}^{n} x_{i} w_{i}\right)
$$

Where:

$\mathrm{X}_{\mathrm{i}}=$ the $\mathrm{i}^{\text {th }}$ input

$\mathrm{w}_{\mathrm{i}}=$ the weight of the link that connect input $\mathrm{i}^{\text {th }}$ with the node

$\mathrm{O}_{\mathrm{j}}=$ output of the $\mathrm{j}^{\text {th }}$ neuron, and

$\mathrm{f}=$ the transfer function

The transfer functions are consider as neuron activation functions based on the characteristic of the study. Sigmoid transfer function is used to avoid large or negative input values that may affect the model. The output of each neuron is calculated by (2).

$$
\frac{1}{1+e^{-x}}
$$

\subsection{Training ANN}

For train the neural network, the database was divided into three samples, 4706 samples (75\%) were randomly selected for training set, 1008 samples (15\%) for validation set, and the residual data (1008 samples) (15\%) were selected for testing data. Shekharan (1998) divided database randomly into 80 percent for training data and 20 percent for testing data in order to train ANN models [11]. The performance of each model is evaluated by Coefficient of Determination $\left(\mathrm{R}^{2}\right)$ by (3).

$$
R^{2}=1-\frac{\sum_{i=1}^{n}\left(A_{i}-P_{i}\right)^{2}}{\sum_{i=1}^{n}\left(A_{i}-\overline{A_{i}}\right)^{2}}
$$

Where:

$P_{i}:$ Predicted values

$A_{i}$ : Observed values

$\overline{A_{i}}:$ Average of observed values

\section{ANALYSIS AND DISCUSSION}

The flexible pavement data was investigated to find the relationship between the structure number and rutting. The other pavement types were not included in this analysis because they do not have sufficient structure data. Before the training process, the data was divided randomly into $70 \%$ for training process and $30 \%$ for validation process. The values of Rutting are plotted against the structural number to show the relationship between them which is not strong as shown in (Fig. 4). 


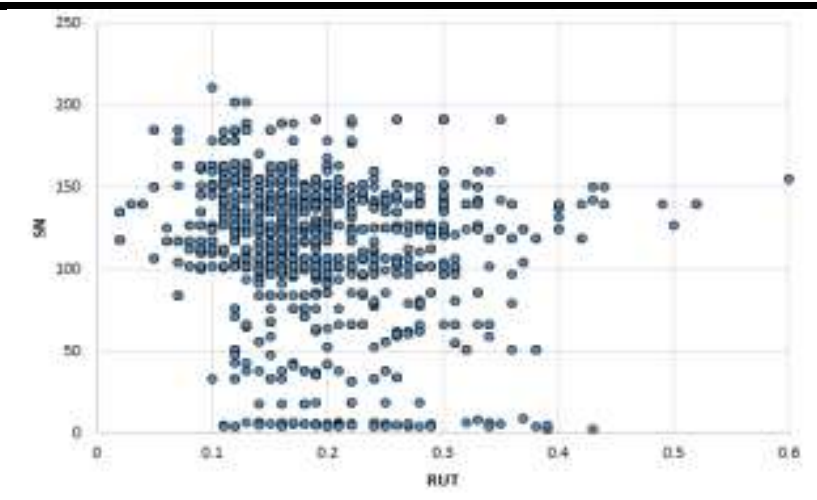

Fig. 4: Scatter plots of SN vs RUT

Different combinations of neural network architecture were examined to develop best ANN models, and finding the correlation between rutting and structure number in asphalt pavements at Iowa's highways. After combining the relevant factors such as structural parameters, traffic loading, subgrade stiffness, and climate parameters. High accuracy estimation was obtained through ANN model with $\mathrm{R}^{2}$ values 0.71 as shown in Figure 5 .

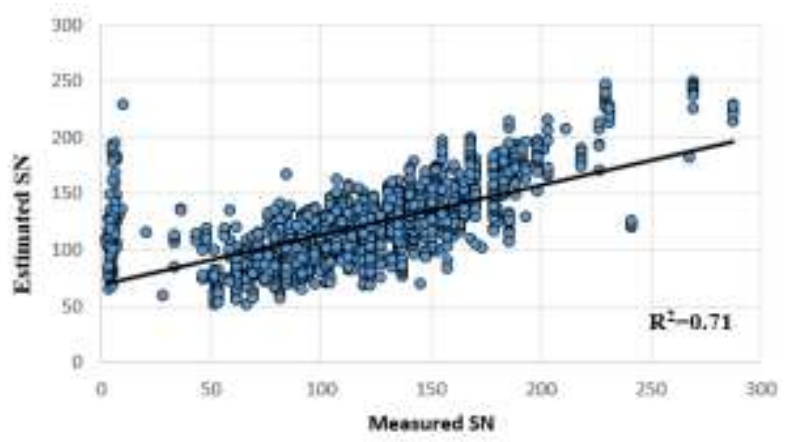

Fig. 5: Fitted line between observed and estimated SN

Finally, predicting the correlation between the functional and structural conditions at network level by utilizing ANN model produces satisfactory results, and the ANN models can deal with noisy data and nonlinear relationship.

\section{CONCLUSION}

Modeling the relationship between the structural capacity and functional conditions is very important to the pavement management system. In this study, the ANN models were used to evaluate the relationship between the functional and structural conditions of existing asphalt pavements at the network level. The IPMP historical data and IEM data were used to train ANN models. The results reported above indicate that it is feasible to use rut depth data as an indicator for structural capacity where structural data is not available or not conducted regularly.

\section{REFERENCES}

[1] AASHTO. 1993. AASHTO Guide for Design of Pavement Structures. AASHTO, Washington, D.C.,.

[2] Adeli, H. 2001. Neural Networks in Civil Engineering: 1989 - 2000. 126 -142. ComputerAided Civil and Infrastructure Engineering.

[3] Agarwal, P., A. Das \& P. Chakroborty. 2006. Simple Model for Structural Evaluation of Asphalt Concrete Pavements at the Network Level. ASCE.

[4] Felker, V., Y. M. Najjar \& M. Hossain. 2004. Modeling The Roughness Progression on Kansas Portland Cement Concrete (PCC) Pavements. 47. Kansas Department of Transportation.

[5] Gencel, O., F. Kocabas, M. S. Gok \& F. Koksal. 2011. Comparison of Artificial Neural Networks and General Linear Model Approaches for the Analysis of Abrasive wear of Concrete. 3486-3494. Construction and Building Materials.

[6] Golshani, N., R. Shabanpour, S. M. Mahmoudifard , S. Derriblr \& A. Mohammadian. 2017. Comparison of Artificial Neural Networks and Statistical CopulaBased Joint Models. 14. Transportation Research Board 96th Annual Meeting.

[7] Hans, N. Z., G. O. Smadi, H. T. Maze, R. R. Souleyrette \& L. J. Resler. July 1997. Iowa's Pavement Management Program Data Base: issues and design consideration." 8th AASHTO/TRB maintenance management conference. In Saratoga Springs, N.Y. Saratoga Springs, N.Y.

[8] Karlaftis, M. G. \& E. I. Vlahogianni. 2011. Statistical Methods versus Neural Networks in Transportation Research: Differences, Similarities and Some Insights. 387-399. Transportation Research Part C.

[9] Noureldin, S., K. Zhu, D. Harris \& S. Li. 2005. NonDestructive Estimation of Pavement Thickness, Structural Number and Subgrade Resilience Along INDOT Highways.

[10] Rafiq, M. Y., G. Bugmann \& D. J. Easterbrook. 2001. Neural Network Design for Engineering Applications. 1541-1552.

[11] Shekharan, A. R. 1998. Effect of Noisy Data on Pavement Performance Prediction by Artificial Neural Networks. 7-13. Transportation Research Record: Journal of the Transportation Research Board.

[12] Simpson, A., G. Rada, B. Visintine, J. Groeger \& J. Guerre. 2013. Improving FHWA's Ability to Assess Highway Infrastructure Health: Development of Next Generation Pavement Performance Measures. FHWA.

[13] Sollazzo, G., T. F. Fwa \& G. Bosurgi. 2017. An ANN model to correlate roughness and structural 
performance in asphalt pavements. 684-693. Construction and Building Matrials.

[14] Vlahogianni, E. I. \& M. G. Karlaftis. 2013. Testing and Comparing Neural Network and Statistical Approaches for Predicting Transportation Time Series. 9-22. Transportation Research Record: Journal of the Transportation Research Board.

[15] Wu, Z., F. Zhou \& S. Hu. 2014. Prediction of Stress Intensity Factors in Pavement Cracking with Neural Networks based on Semi-Analytical FEA. 10211030. Expert Systems with Applications.

[16] Zhang, G., B. E. Patuwo \& M. Y. Hu. 1998. Forcasting with artificial neural networks: The state of the art. 35-62. International Journal of Forecasting. 